\title{
O efeito da impulsividade, autoaversão e autocompaixão nos traços borderline na adolescência: Estudo das diferenças entre sexos
}

\author{
The effect of impulsivity, self-disgust and self-compassion in borderline \\ features in adolescence: Study of sex differences
}

\author{
Diogo Carreiras (1) \\ Paula Castilho (1) \\ Marina Cunha $(1,2)$ \\ (1) Univ Coimbra, CINEICC, FPCEUC, Portugal \\ (2) Inst Sup Miguel Torga, Coimbra, Portugal
}

Recebido: 31/03/2020; Revisto: 26/04/2020; Aceite: 18/05/2020.

https://doi.org/10.31211/rpics.2020.6.1.170

\section{Resumo}

Contexto: A adolescência é uma etapa desenvolvimental com mudanças biológicas, psicológicas e sociais que irão influenciar o funcionamento na idade adulta. A investigação em torno das Perturbações da Personalidade, e em particular da Perturbação Borderline da Personalidade (PBP), tem cada vez mais investido no estudo de traços disfuncionais e inflexíveis em idades precoces, uma vez que é claro que uma Perturbação da Personalidade não se manifesta apenas subitamente na idade adulta. Existe uma trajetória desenvolvimental que deve ser melhor compreendida e explorada. Objetivo: Neste sentido, o presente trabalho teve como objetivo analisar o contributo de processos e mecanismos psicológicos, como a impulsividade, autoaversão e autocompaixão, para a compreensão dos traços borderline na adolescência. Métodos: Este estudo tem um desenho transversal e uma amostra constituída por 440 adolescentes da população geral (278 raparigas e 162 rapazes), com idades compreendidas entre os 14 e os 17 anos. Com recurso ao SPSS, realizaram-se testes $t$ para amostras independentes, correlações de Pearson e regressões lineares. Resultados: As raparigas, quando comparadas com os rapazes, apresentaram níveis mais elevados de autoaversão, depressão e traços borderline e níveis mais baixos de autocompaixão. Os modelos de regressão hierárquica para testar o poder preditivo da impulsividade, autoaversão e autocompaixão nos traços borderline foram significativos, explicando $46 \%$ da variância dos traços borderline em rapazes e $58 \%$ nas raparigas, controlando o efeito da depressão. Enquanto que nas raparigas, todas as variáveis apresentaram um contributo significativo (depressão, impulsividade, autocompaixão e autoaversão), nos rapazes apenas a depressão, impulsividade e autocompaixão revelaram poder preditivo. Conclusões: Os dados desta investigação salientam variáveis essenciais para compreender os traços borderline em adolescentes, bem como as diferenças nesses mecanismos psicológicos entre raparigas e rapazes, tendo significativas implicações para a investigação e, sobretudo, para a prática clínica e prevenção.
\end{abstract}

Palavras-Chave: Adolescência; Autoaversão; Autocompaixão; Impulsividade; Traços Borderline da Personalidade.

$\begin{array}{ll}\text { DI\&D | ISMT } & \text { Publicação em Acesso Aberto } \\ \text { rpics@ismt.pt } & \text { (c)2020. O(s) Autor(es). Este é um artigo de acesso aberto } \\ \text { distribuído sob a Licença Creative Commons Attribution, } & \text { que permite uso, distribuição e reprodução sem restrições } \\ \text { https://rpics.ismt.pt } & \begin{array}{l}\text { em qualquer meio, desde que o trabalho original seja } \\ \text { devidamente citado. }\end{array}\end{array}$

Diogo Carreiras

CINEICC, Centro de Investigação em Neuropsicologia e Intervenção Cognitivo Comportamental, Universidade de Coimbra. R. Colégio Novo, 3000-115 Coimbra, Portugal E-mail: diogocarreiras1@gmail.com 


\begin{abstract}
Background: Adolescence is a developmental stage with biological, psychological, and social changes that will influence the individual functioning in adulthood. Recently, research on borderline personality disorder (BPD) has been focusing on dysfunctional and inflexible features in early ages, since a personality disorder does not appear suddenly in adulthood. The developmental path should be better understood and explored. Objective: Accordingly, the current study aimed at analyzing the contribution of psychological processes, specifically impulsivity, self-disgust, and self-compassion, for understanding borderline features in adolescence. Methods: This study had a cross-sectional design and a sample of 440 adolescents from the general population ( 278 girls and 162 boys), with ages ranging between 14 and 17 years. In SPSS, we conducted Student's $t$-tests, Pearson correlations, and linear regressions. Results: Girls presented higher levels of self-disgust, and borderline features in comparison with boys and lower levels of self-compassion. Regression models to test the predictive value of impulsivity, self-disgust, and selfcompassion on borderline features were significant. The model explained $43 \%$ of borderline features for boys and $57 \%$ for girls. For girls, all variables (impulsivity, self-compassion, and self-disgust) presented a significant contribution, and for boys, only impulsivity and self-compassion were significant predictors. Conclusions: These results added evidence of important variables to understand better borderline features in adolescents and identified sex differences in these psychological mechanisms. This study has important implications for research, clinical practice, and prevention.
\end{abstract}

Keywords: Adolescence; Borderline features; Impulsivity; Self-compassion; Self-disgust.

\title{
Introdução
}

Descrita como incapacitante e interferente, a perturbação borderline da personalidade (PBP) é caracterizada por um padrão persistente de impulsividade, instabilidade na autoimagem, no afeto e nas relações interpessoais e no comportamento, bem como por marcadas dificuldades de regulação emocional (American Psychiatric Association [APA], 2013; Leichsenring et al., 2011). A prevalência da PBP na população geral situase entre $1,6 \%$ e $5,9 \%$ (APA, 2013).

Embora habitualmente a incidência seja estudada na população adulta, a PBP pode ser diagnosticada na adolescência, quando tal for justificado (APA, 2013). A investigação em torno dos traços borderline na adolescência tem crescido nos últimos anos, partindo da premissa de que, sendo a PBP uma patologia da personalidade, existe uma trajetória desenvolvimental disfuncional que pode ser detetada em faixas etárias mais jovens (Paris, 2009). Vários autores salientam que padrões comportamentais, cognitivos e afetivos disfuncionais manifestam-se antes dos 18 anos de idade e, portanto, traços ou características borderline podem ser identificadas na adolescência (Bradley et al., 2005; Crick et al., 2005; Sharp \& Bleiberg, 2007). De facto, Zanarini et al. (2006) referem que pessoas com traços borderline reconhecem o início dos seus sintomas por volta dos 11 anos ( $D P=5$ anos) e que receberam tratamento para essas dificuldades, pela primeira vez, em média aos 17 anos $(D P=6$ anos). A prevalência de PBP na população adolescente é semelhante à prevalência na população adulta, entre 1,3\% e 1,6\% (Johnson et al., 2008; Lewinsohn et al., 1997), no entanto, em contexto hospitalar, esta prevalência aumenta para os 22\% (Chanen et al., 2008). Quanto a diferenças entre sexos, os resultados não são consensuais pois alguns estudos apontam para a existência de traços borderline mais elevados nas mulheres (Swartz et al., 1990; Trull et al., 2010), ao passo que outros estudos não reportam diferenças significativas entre sexos (Aragonès et al., 2013; Morey et al., 2002).

Como referido anteriormente, a impulsividade é uma característica central na PBP. Chapman et al. (2008) demonstraram que as pessoas com mais características borderline apresentam níveis de impulsividade significativamente superiores a outras com menos características borderline. Com frequência, a impulsividade nas 
pessoas com traços borderline mais elevados está associada a consequências negativas, como comportamentos autolesivos (Brown et al., 2002; Plener et al., 2015), abuso de substâncias ou comportamento sexual de risco (Sebastian et al., 2013). Fossati et al. (2014) estudaram a impulsividade, regulação emocional e traços borderline em 1157 adolescentes, comparando três grupos distintos em função do nível de traços borderline (alto, médio e baixo). Concluíram que o grupo com um nível elevado de traços borderline se diferenciava dos outros grupos, revelando uma tendência significativamente maior para ser impulsivo.

Adicionalmente, alguns estudos encontraram uma relação significativa e positiva entre a PBP e a autoaversão (Guiomar, 2015; Ille et al., 2014; Schienle et al., 2013). A autoaversão tem sido descrita como a experiência autoconsciente da emoção básica de nojo orientada para o "eu" (Overton et al., 2008; Power \& Dalgleish, 2008). É uma abstração generalizada e disfuncional da aversão a aspetos pessoais, internos e externos, relativamente estáveis e duradouros (Olatunji et al., 2012; Powell et al., 2015). Sendo o nojo/aversão uma emoção básica, a autoaversão apresenta diferentes componentes: uma cognitiva/emocional, outra fisiológica e uma componente comportamental, que inclui afastamento e fuga (Carreiras, 2014; Ekman, 1992; Overton et al., 2008; Powell et al., 2015). Estudos têm mostrado uma associação positiva entre a autoaversão e sintomas de depressão, ansiedade e ideação suicida (Carreiras, 2014; Overton et al., 2008), bem como perturbações do comportamento alimentares (Ille et al., 2014). Em adolescentes da comunidade geral, a investigação de Guilherme (2019) apresentou correlações positivas entre a autoaversão e sintomas psicopatológicos, impulso, autodano e ideação suicida e uma associação negativa entre a autoaversão e autocompaixão.

Uma alternativa à autoaversão é a capacidade de autotranquilização e de se ser gentil e bondoso consigo mesmo, nomeadamente quando se está em sofrimento físico e psicológico. A autocompaixão revela-se um processo psicológico protetor cada vez mais estudado e que pressupõe ser sensível ao sofrimento do próprio/a, com uma motivação genuína para o aliviar esse sofrimento em situações difíceis. Incluindo um conjunto de atitudes essenciais, a autocompaixão consiste em não ajuizar e rotular, compreender o sofrimento como parte de uma experiência humana comum, partilhada com todos os seres humanos, e ter a capacidade de estar com esse sofrimento sem o tentar suprimir ou evitar (Neff, 2003, 2016). Portanto, as estratégias de autocompaixão relacionam-se com o sistema de vinculação e segurança e traduzem-se num estado interno de calma, comportamentos ativos de exploração, criatividade, afiliação e cuidado pelo eu. Diversos e studos têm mostrado associações negativas entre a autocompaixão e a psicopatologia (Krieger et al., 2013; Marsh et al., 2018) e correlações positivas entre a autocompaixão e o bem-estar e funcionamento psicológico adaptativo (Bluth et al., 2017; Neff et al., 2007). Em adultos, existem alguns estudos que mostraram o papel protetor da autocompaixão relativamente à PBP (Keng \& Wong, 2017; Loess, 2015; Scheibner et al., 2018; Warren, 2015), no entanto, no que toca à população adolescente, estudos sobre os traços borderline e a sua relação com a autocompaixão são escassos. Não obstante, estudos com adolescentes já apresentaram evidência de que ser autocompassivo está associado a outcomes psicológicos positivos (Bluth \& Balton, 2015; Cunha et al., 2013, 2016). Adicionalmente, diferenças na autocompaixão entre rapazes e raparigas foram previamente reportadas, com as raparigas a apresentarem níveis mais baixos, especialmente as mais velhas (Bluth et al., 2017). Numa meta-análise, Yarnell et al. (2015) reportaram a evidência de que pessoas do sexo masculino apresentarem níveis de autocompaixão ligeiramente superiores às do sexo feminino.

Considerando as evidências dos estudos previamente descritos, e dada a falta de investigação em Portugal na área dos traços borderline na adolescência e de fatores que contribuem para a sua manutenção, o principal objetivo deste trabalho foi estudar o contributo de variáveis relacionadas com a experiência do self para a compreensão 
dos traços borderline na adolescência. Com base na revisão da literatura, este será o primeiro estudo português a explorar estas variáveis e a sua relação com os traços borderline, testando diferenças entre os sexos. Assim, pretendemos analisar o contributo da impulsividade, da autoaversão e da autocompaixão nos traços borderline em adolescentes e explorar a possível existência de diferenças nestas variáveis entre rapazes e raparigas. Esperamos que a autoaversão, a impulsividade e a autocompaixão tenham um contributo individual único na explicação dos traços borderline nos adolescentes, controlado o efeito dos sintomas depressivos. Esperamos também encontrar diferenças entre os sexos, nomeadamente níveis mais elevados de sintomas depressivos, traços borderline e autoaversão nas raparigas, e níveis mais elevados de autocompaixão nos rapazes.

\section{Método}

\section{Participantes}

A amostra do presente estudo foi composta por 440 adolescentes portugueses da população geral, dos quais 278 (63\%) são do sexo feminino e 162 (37\%) do sexo masculino, com idades compreendidas entre os 14 e os 17 anos, $M=15,47 ; D P=0,83$. Os adolescentes eram estudantes do ensino básico e secundário dos quais setenta e cinto (17\%) estavam no 9o ano, duzentos e seis (47\%) no 10 ano e cento e cinquenta e nove (36\%) no 11 o ano de escolaridade. Não foram encontradas diferenças significativas entre rapazes e raparigas relativamente à idade, $t(210)=0,14, p=0,89$, e anos de escolaridade, $t(210)=0,83, p=0,41$.

\section{Instrumentos}

Escala de Traços de Personalidade Borderline para Adolescentes (ETPB-A; Borderline Personality Features Scale for Children - BPFS-C; Sharp et al., 2014; versão portuguesa: Carreiras et al., 2020). Este instrumento de autorrelato é unidimensional e constituído por 10 itens, que avaliam os traços borderline de adolescentes, com questões sobre a forma como o sujeito se sente em relação a si próprio e aos outros (e.g., "A maneira como me sinto muda muito.", "Sinto que há algo importante que falta em mim, mas não sei o que é."). Os itens são cotados numa escala de Likert de 5 pontos ( 1 = "Nunca verdadeiro"; 5 = "Sempre verdadeiro"). Quanto maior o somatório das pontuações de todos os itens, maiores os níveis de traços borderline. No estudo da versão original, a BPFS-C apresentou boa consistência interna ( $\alpha$ de Cronbach = 0,85, Sharp et al., 2014), bem como no estudo da versão portuguesa ( $\alpha$ de Cronbach $=0,77$, Carreiras et al., 2020). No presente estudo, o alfa de Cronbach foi de 0,84 .

Escala de Autocompaixão para Adolescentes (EAC-A; Self-Compassion Scale - SCS; Neff, 2003; versão portuguesa para adolescentes: Cunha et al., 2016). Esta escala destina-se a avaliar a autocompaixão em adolescentes, ou seja, a capacidade de serem calorosos, aceitantes e compreensivos consigo próprios em momentos difíceis e de sofrimento (e.g., "Quando as coisas me correm mal, vejo as dificuldades como fazendo parte da vida, e pelas quais toda a gente passa.", "Quando passo por tempos difíceis tenho tendência a ser muito exigente e duro/a comigo mesmo/a."). É composta por 26 itens que compõem seis subescalas (calor/compreensão, isolamento, humanidade comum, autojulgamento, mindfulness e sobreidentificação), cotados numa escala de Likert de 5 pontos ( 1 = "Quase nunca"; 5 = "Quase sempre"). 0 total da escala é uma média das pontuações das subescalas, após inverter as pontuações dos itens das subescalas isolamento, autojulgamento" e sobreidentificação. Resultados mais elevados representam níveis mais elevados de 
autocompaixão. A EAC-A revelou boa consistência interna no estudo original ( $\alpha$ de Cronbach $=0,92$; Neff, 2003) e na versão portuguesa para adolescentes ( $\alpha$ de Cronbach $=0,85$, Cunha et al., 2016). No presente estudo, o alfa de Cronbach da escala total foi de 0,84.

Escala Multidimensional da Autoaversão para adolescentes (EMA-A; Escala Multidimensional da Autoaversão EMA; Carreiras, 2014; versão para adolescentes: Guilherme et al., 2020). Esta escala portuguesa avalia a autoaversão, ou seja, a emoção de aversão/nojo direcionada para aspetos internos e externos do "eu". É constituída por 30 itens que compõem quatro subescalas: ativação defensiva (componente fisiológica da emoção; e.g., "Quando sinto aversão em relação a mim, a minha respiração fica acelerada."), cognitivo-emocional (pensamentos e sentimentos que refletem a relação de hostilidade e aversão para com o "eu"; e.g., "Quando sinto aversão em relação a mim, sinto-me diminuído/a, inferior e pequeno/a."), evitamento (comportamentos destinados a esconder ou evitar esses aspetos considerados aversivos"; e.g., "Quando sinto aversão em relação a mim, desvio o olhar do meu corpo.") e exclusão (comportamentos para excluir ou eliminar os aspetos do "eu" considerados tóxicos"; e. g., "Quando sinto aversão em relação a mim, sinto vontade de cortar, queimar, eliminar essa parte de mim mesmo/a."). Os itens são cotados numa escala de Likert de cinco pontos ( 1 = "Nunca"; 5 = "Sempre") e depois feito o somatório das pontuações dos itens. Maiores pontuações representam maiores níveis de autoaversão. A consistência interna da versão original para adolescentes é muito boa ( $\alpha$ de Cronbach $=0,97$; Guilherme et al., 2020), tal como no presente estudo ( $\alpha$ de Cronbach $=0,96$ ).

Questionário de Impulso, Autodano e Ideação Suicida para Adolescentes (QIAIS-A; Carvalho et al., 2015). O referido instrumento de autorrelato é composto por 58 itens que avaliam o impulso/impulsividade, comportamentos autolesivos, motivos e funções desses comportamentos, e ideação suicida. Os itens agrupam-se em quatro subescalas: impulso (8 itens), autodano (14 itens), funções do autodano (31 itens) e ideação suicida (3 itens) e são cotados numa escala de Likert de quatro pontos ( $0=$ "Nunca acontece comigo"; 3 = "Acontece-me sempre"). Para este estudo, apenas recorremos à subescala impulso (e.g., "Faço coisas sem pensar nas consequências.") através do somatório das pontuações dos 8 itens, em que valores mais elevados significam maior impulsividade. Esta subescala apresentou uma consistência interna adequada neste estudo ( $\alpha$ de Cronbach $=$ 0,78 ), semelhante à encontrada no estudo da versão original ( $\alpha$ de Cronbach $=0,76$; Carvalho et al., 2015).

Escala de Ansiedade Depressão e Stress para adolescentes (DASS-21-A; Lovibond \& Lovibond, 1995; versão portuguesa para adolescentes: Pais-Ribeiro et al., 2004). Este instrumento de autorrelato é composto por 21 itens que avaliam sintomas de depressão, ansiedade e stress. Os itens agrupam-se em três subescalas: depressão ( 7 itens), ansiedade (7 itens) e stress (7 itens) e são cotados numa escala de Likert de quatro pontos ( 0 = "Não se aplicou nada a mim"; 3 = "Aplicou-se a mim a maior parte das vezes"). Para este estudo, apenas recorremos à subescala "Depressão" (e.g., "Não consegui sentir nenhum sentimento positivo") através do somatório das pontuações dos sete itens, em que valores mais elevados significam maior sintomatologia depressiva. Neste estudo, esta subescala apresentou uma consistência interna bastante adequada ( $\alpha$ de Cronbach $=0,88$ ), semelhante à encontrada no estudo da versão original ( $\alpha$ de Cronbach $=0,91$; Lovibond \& Lovibond, 1995) e versão portuguesa ( $\alpha$ de Cronbach = 0,91; Pais-Ribeiro et al., 2004).

\section{Procedimentos}

O presente estudo faz parte de uma investigação mais alargada, cujos procedimentos foram aprovados pela Direção Geral de Educação (DGE) do Ministério da Educação, pela Comissão Nacional de Proteção de Dados (CNPD; autorização n. 6713/ 2018) e pelos órgãos diretivos dos estabelecimentos de ensino. Posteriormente, foram 
agendadas com o Investigador responsável as datas para a recolha de dados, que se realizou nos estabelecimentos de ensino em horário escolar. Os consentimentos informados, com informação sobre o estudo, objetivos, proteção de dados, confidencialidade e anonimato foram enviados para os encarregados de educação. Os instrumentos de autorrelato foram administrados aos adolescentes, cujos encarregados de educação concederam permissão. Os jovens frequentavam estabelecimentos de ensino básico e secundário e os dados foram recolhidos entre outubro de 2018 e março de 2019, no centro e norte de Portugal Continental. Durante o preenchimento dos questionários, o investigador e o professor responsável pela unidade curricular estiveram presentes para esclarecer dúvidas e garantir a independência das respostas.

\section{Análise Estatística}

Para a análise dos dados, utilizámos o software IBM SPSS versão 23. Estatísticas descritivas e testes $t$ de Student para amostras independentes foram realizados para examinar as variáveis sociodemográficas e explorar diferenças entre sexos, respetivamente. As associações entre as variáveis foram analisadas através do cálculo do coeficiente de correlação de Pearson. De acordo com Dancey e Reidy (2017), valores entre 0,10 e 0,39 são considerados fracos, entre 0,40 e 0,69, moderados e acima de 0,70, fortes. 0 tamanho do efeito foi calculado de acordo com Cohen (1988), considerando pequenos valores de $d$ entre 0,20 e 0,49, médios entre 0,50 e 0,79 e elevados acima de 0,80. Regressões múltiplas foram realizadas para explorar o poder preditivo das variáveis independentes (impulsividade, autoaversão, autocompaixão e depressão) sobre a variável dependente (traços borderline). A independência dos erros foi analisada com recurso aos valores de Durbin-Watson, considerando valores abaixo de 2,5 aceitáveis. A multicolinearidade e singularidade das variáveis foi examinada através dos Variance Inflation Fator (VIF), sendo aceitáveis valores inferiores a 5 (Kline, 2005).

\section{Resultados}

\section{Análise preliminar dos dados}

Não foram encontradas violações severas à normalidade dos dados após analisar os valores de assimetria (|Sk|< 3) e curtose $(|K u|<8)$. Os valores de Durbin-Watson foram aceitáveis (entre 1,65 e 1,82). Os VIF foram abaixo do valor recomendado de 5 . No geral, as análises preliminares evidenciaram um ajustamento adequado dos dados para prosseguir com as análises estatísticas.

\section{Análise descritiva e diferenças entre sexos}

Na Tabela 1 são apresentadas as estatísticas descritivas das variáveis em estudo para a amostra total e por sexo. Comparativamente aos rapazes, as raparigas apresentaram níveis mais elevados de traços borderline, $t(438)=2,25, p=0,03$, e depressão, $t_{(438)}=2,25, p=0,02$, com um tamanho de efeito pequeno, bem como níveis mais elevados de autoaversão, $t(438)=5,42, p<0,001$, com tamanho de efeito médio. Quanto à autocompaixão, os rapazes apresentam níveis mais elevados, $t(438)=3,50, p<0,001$, com um tamanho de efeito pequeno. Não foram encontradas diferenças estatisticamente significativas entre os sexos relativamente à impulsividade. 


\section{Tabela 1}

Médias (M), Desvios-Padrão (DP), Testes t para Amostras Independentes (t) e Tamanho do Efeito (d) para as Diferentes Variáveis em Estudo na Amostra Total e na Amostra Dividida por Sexos

\begin{tabular}{lccccc}
\hline \multirow{2}{*}{ Variáveis } & $\begin{array}{c}\text { Total } \\
(N=440)\end{array}$ & $\begin{array}{c}\text { Rapazes } \\
(n=162)\end{array}$ & $\begin{array}{c}\text { Raparigas } \\
(n=278)\end{array}$ & $t(g /)$ & $d$ de Cohen \\
\cline { 2 - 4 } & $M(S D)$ & $M(S D)$ & $M(S D)$ & & 0,22 \\
\hline Traços borderline (ETPB-A) & $25,45(7,35)$ & $24,41(7,44)$ & $26,05(7,25)$ & $2,25 *(438)$ & 0,05 \\
Impulsividade (QIAIS-A) & $7,16(4,27)$ & $7,30(4,25)$ & $7,08(4,29)$ & $0,51(438)$ & 0,56 \\
Autoaversão (EMA-A) & $18,65(21,19)$ & $11,69(16,78)$ & $22,70(22,43)$ & $5,42 * *(438)$ & 0,35 \\
Autocompaixão (SCS-A) & $3,13(0,64)$ & $3,27(0,56)$ & $3,05(0,68)$ & $3,50 * *(438)$ & 0,24 \\
Depressão (EADS-21) & $5,15(4,75)$ & $4,43(4,42)$ & $5,57(4,89)$ & $2,45 *(438)$ & 0,24
\end{tabular}

Nota . ETPB-A = Escala de Traços de Personalidade Borderline para Adolescentes; EAC-A = Escala de Autocompaixão para Adolescentes; EMA-A = Escala de Autoaversão para Adolescentes; QIAIS-A: Questionário de Impulso, Autodano e Ideação Suicida para Adolescentes; EADS-21-A = Escala de Ansiedade Depressão e Stress para Adolescentes.

${ }^{*} p<0,05 ;{ }^{*} p<0,001$.

\section{Correlações entre traços borderline, impulsividade, autoaversão, autocompaixão e depressão}

Como apresentado na Tabela 2, encontramos correlações negativas e moderadas entre a autocompaixão e os traços borderline, sintomas depressivos e impulsividade, o que significa que elevada autocompaixão está associada a menores níveis de traços borderline, sintomas depressivos e impulsividade. Encontramos também correlações positivas moderadas e fortes entre a impulsividade, a autoaversão, os sintomas depressivos e os traços borderline, ou seja, elevados traços borderline estão associados a maiores níveis de impulsividade, de sintomatologia depressiva e autoaversão. Todos os coeficientes de correlações foram significativos ao nível de $p<0,001$.

\section{Tabela 2}

Correlações de Pearson entre as Variáveis em Estudo $(N=440)$

\begin{tabular}{|c|c|c|c|c|c|}
\hline & 1 & 2 & 3 & 4 & 5 \\
\hline 1. Traços borderline (ETPB-A) & - & - & - & - & 一 \\
\hline 2. Impulsividade (QIAIS-A) & $0,60 * *$ & - & - & - & - \\
\hline 3. Autoaversão (EMA-A) & $0,56 * *$ & $0,43^{* *}$ & - & - & - \\
\hline 4. Autocompaixão (EAC-A) & $-0,58 * *$ & $-0,39 * *$ & $-0,63 * *$ & - & - \\
\hline 5. Depressão (EADS-21-A) & $0,56 * *$ & $0,44 * *$ & $0,66 * *$ & $-0,67 * *$ & 一 \\
\hline
\end{tabular}

Nota . ETPB-A = Escala de Traços de Personalidade Borderline para Adolescentes; EAC-A = Escala de Autocompaixão para Adolescentes; EMA-A = Escala de Autoaversão para Adolescentes; QIAIS-A: Questionário de Impulso, Autodano e Ideação Suicida para Adolescentes; EADS-21-A = Escala de Ansiedade Depressão e Stress para Adolescentes.

$* p<0,05 ; * *<0,001$.

\section{Regressões múltiplas na explicação dos traços borderline para ambos os sexos}

Dadas as diferenças encontradas entre rapazes e raparigas nas variáveis em estudo, foram realizadas duas regressões múltiplas, uma para cada grupo. Em ambas as regressões, a variável dependente foi os traços borderline e as variáveis 
independentes foram a depressão, impulsividade, autoaversão, autocompaixão. Nos rapazes, o modelo final (Tabela 3) foi significativo e explicou $46 \%$ da variância dos traços borderline, $F(4,157)=34,92, p<0,001$. Dos preditores testados, a impulsividade, $b=0,35, p<0,001$, e a autocompaixão, $b=-0,25, p=0,004$, revelaram significância estatística, bem como a depressão, $\theta=0,27, p=0,002$. A autoaversão não se revelou um preditor significativo, $\theta=0,05, p=0,58$. Para os rapazes, menor autocompaixão e maior impulsividade relacionaram-se com níveis mais elevados de traços borderline.

\section{Tabela 3}

Regressão Múltipla a Explicar os Traços Borderline em Adolescentes do Sexo Masculino (N = 162)

\begin{tabular}{lcccc}
\hline Variáveis & $R_{2}$ & R2 ajustado & $B$ & Erro \\
\hline & 0,47 & 0,46 & & $\beta$ \\
Depressão (EADS-21-A) & & 0,46 & 0,15 & $0,27^{*}$ \\
Impulsividade (QIAIS-A) & & 0,62 & 0,12 & $0,35^{* *}$ \\
Autoaversão (EMA-A) & & 0,02 & 0,04 & 0,06 \\
Autocompaixão (EAC-A) & & $-3,29$ & 1,13 & $-0,27^{*}$ \\
\hline
\end{tabular}

Nota. ETPB-A = Escala de Traços de Personalidade Borderline para Adolescentes; EAC-A = Escala de Autocompaixão para Adolescentes; EMA-A = Escala de Autoaversão para Adolescentes; QIAIS-A = Questionário de Impulso, Autodano e Ideação Suicida para Adolescentes; EADS-21-A = Escala de Ansiedade Depressão e Stress para Adolescentes.

${ }^{*} p<0,05 ; * p<0,001$.

Relativamente ao grupo constituído apenas por sujeitos do sexo feminino, o mesmo modelo foi testado (Tabela 4). Neste caso, a variância dos traços borderline foi explicada em $58 \%$, num modelo estatisticamente significativo, $F(4,273)=$ 96,24, $p<0,001$. Todos os preditores demonstraram significância estatística: impulsividade, $b=0,38, p<0,001$, autoaversão, $b=0,26, p=0,002$, autocompaixão, $b=-0,23, p<0,001$, e depressão, $b=0,17, p=0,003$. Para as raparigas, menor autocompaixão, maior impulsividade e maior autoaversão relacionam-se com níveis mais elevados de traços borderline.

\section{Tabela 4}

Regressão Múltipla a Explicar os Traços Borderline em Adolescentes do Sexo Feminino (N = 278)

\begin{tabular}{lcccc}
\hline Variáveis & $R_{2}$ & R2 ajustado & $B$ & Erro \\
\hline & 0,59 & 0,58 & & \\
Depressão (EADS-21-A) & & 0,25 & 0,08 & $0,17^{*}$ \\
Impulsividade (QIAIS-A) & & 0,64 & 0,08 & $0,38^{* *}$ \\
Autoaversão (EMA-A) & & 0,06 & 0,02 & $0,18^{*}$ \\
Autocompaixão (EAC-A) & & $-2,44$ & 0,56 & $-0,23^{* *}$ \\
\hline
\end{tabular}

Nota . ETPB-A = Escala de Traços de Personalidade Borderline para Adolescentes; EAC-A = Escala de Autocompaixão para Adolescentes; EMA-A = Escala de Autoaversão para Adolescentes; QIAIS-A = Questionário de Impulso, Autodano e Ideação Suicida para Adolescentes; EADS-21-A = Escala de Ansiedade Depressão e Stress para Adolescentes.

${ }^{*} p<0,05 ; * p<0,001$. 


\section{Discussão}

O número de estudos sobre traços borderline na adolescência tem crescido nos últimos anos. Tal evidência está relacionada com alguns fatores, como o reconhecimento da trajetória desenvolvimental da PBP, e com o facto de o início dos sintomas borderline e da procura de tratamento serem reportados em idades precoces (Crick et al., 2005; Paris, 2009; Zanarini et al., 2006). Porém, em Portugal, verifica-se ainda uma lacuna na investigação deste tema, pelo que o presente estudo teve dois grandes objetivos: explorar o poder preditivo da impulsividade, da autoaversão e da autocompaixão relativamente aos traços borderline, controlando a sintomatologia depressiva; e analisar possíveis diferenças entre adolescentes do sexo feminino e masculino nessas variáveis.

Foram encontradas diferenças entre rapazes e raparigas em algumas variáveis em estudo. Os dados mostraram que os traços borderline nas raparigas revelaram-se significativamente mais elevados, comparativamente aos rapazes, indo ao encontro de estudos anteriores (Swartz et al., 1990; Trull et al., 2010), o que é também consistente com o facto de a PBP ser predominantemente diagnosticada em mulheres (APA, 2013). Do ponto de vista da cultura ocidental, características borderline como emocionalidade intensa, sentimentos de dependência e abandono estão mais associadas ao sexo feminino, o que pode conduzir a uma cotação mais elevada destes sintomas pelas raparigas e explicar os resultados obtidos neste e noutros estudos. O mesmo padrão foi encontrado para a autoaversão, com as raparigas a revelarem níveis mais elevados, o que vai ao encontro de estudos anteriores (Guilherme et al., 2020). As jovens parecem ter uma relação mais negativa e crítica consigo próprias, caraterizada por pensamentos e sentimentos de aversão e repulsa em relação a aspetos físicos e de personalidade. O que poderá relacionar-se com uma maior sensibilidade das adolescentes aos sinais de aprovação e desaprovação dos outros, um aspeto central nesta fase de desenvolvimento na formação da identidade.

Relativamente à autocompaixão, os resultados evidenciaram um padrão oposto, já que foram os adolescentes do sexo masculino que se mostraram mais compassivos consigo próprios, reportando, em momentos de sofrimento, uma maior capacidade de autotranquilização, menos enredamento na experiência interna e uma vivência de conectividade e de humanidade comum. Outros estudos, portugueses e internacionais, tinham previamente comprovado que os rapazes tendem a apresentar níveis mais elevados de autocompaixão em comparação com as raparigas (Bluth et al., 2017; Cunha et al., 2016; Xavier et al., 2016; Yarnell et al., 2015). Uma das possíveis explicações para os níveis mais elevados de autocompaixão no sexo masculino será o facto de os homens não se enredarem e sobreidentificarem de forma excessiva com pensamentos e experiência interna negativa, em comparação com as mulheres. Ademais, as raparigas tendem a ser mais autocríticas e mais predispostas a terem um discurso interno negativo (Yarnell et al., 2015).

Quanto à impulsividade, não encontrámos diferenças significativas entre os sexos embora a literatura aponte para níveis mais elevados em pessoas do sexo masculino (Cross et al., 2011). Consideramos que a ausência de diferenças se relaciona com a utilização de uma medida que é uma subescala unidimensional com apenas oito itens e não um instrumento destinado a avaliar a impulsividade. Como tal, possivelmente esta subescala não capta nuances particulares e mais específicas da impulsividade que permitem diferenciar a sua expressão em função do sexo. No futuro, é recomendável que outros estudos utilizem medidas mais completas de avaliação da impulsividade.

Todas as variáveis em estudo revelaram-se significativamente associadas entre si. Especificamente, foi encontrada uma correlação positiva e forte entre a impulsividade e os traços borderline, como já confirmado em estudos prévios (Chapman et al., 2008; Fossati et al., 2014). A autoaversão também estava positivamente correlacionada 
com os traços borderline, depressão e com a impulsividade, reforçando estudos anteriores que evidenciaram a relação entre a aversão direcionada para aspetos do eu e sintomas psicológicos negativos (Carreiras, 2014; Overton et al., 2008), inclusivamente da PBP (Ille et al., 2014). Os valores da autocompaixão nos adolescentes mostraram-se negativamente correlacionados com a impulsividade, a autoaversão, os traços borderline e sintomatologia depressiva, indicando o potencial efeito protetor da capacidade genuína de ser sensível ao próprio sofrimento, reconhecendo-o, e de agir no sentido de o aliviar, no desenvolvimento de outcomes psicológicos negativos, como ansiedade, depressão e stress (Krieger et al., 2013; Marsh et al., 2018).

Quanto ao conjunto de variáveis que melhor explica os traços borderline, os modelos de regressão múltipla, conduzidos para rapazes e raparigas, dadas as diferenças entre os sexos reportadas anteriormente, revelaram resultados importantes para a compreensão da forma como essas variáveis se comportam, em função do sexo. Em primeiro lugar, ambos os modelos foram significativos, explicando percentagens consideráveis dos traços borderline: $46 \%$ para os rapazes e 58\% para as raparigas. Em segundo lugar, podemos concluir que, independentemente do sexo e dos níveis de sintomatologia depressiva, a impulsividade e a autocompaixão são variáveis importantes a considerar na compreensão dos traços borderline nestas faixas etárias. Contudo, em terceiro lugar, os resultados mostraram que a autoaversão apenas foi um preditor significativo para os traços borderline nas raparigas, o que aponta para uma maior sensibilidade das pessoas do sexo feminino à relação negativa e de aversão por aspetos do eu, internos e/ou externos, com elevado autocriticismo, na explicação dos traços borderline. No grupo composto por sujeitos do sexo masculino, a autoaversão não teve um contributo único e independente.

Os resultados obtidos permitem refletir sobre importantes implicações clínicas. Os traços borderline estão presentes nesta faixa etária, em rapazes e raparigas, e estão associados a variáveis psicológicas negativas, como a impulsividade, a autoaversão e a depressão. Para os rapazes, a autoaversão não parece ser um aspeto relevante na explicação da variância dos traços borderline, enquanto para as raparigas desempenha um papel significativo. Como tal, intervenções individuais ou grupais com as raparigas com traços borderline marcados deverão trabalhar o desenvolvimento de uma relação mais calorosa e aceitante do eu como forma de atenuar esses traços. Krawitz (2012) defendeu que a abordar e desenvolver autocompaixão seria uma intervenção promissora no tratamento crónico de autoaversão em pessoas com PBP. Assim, autocompaixão pode funcionar como um fator protetor, estando negativamente associada com os traços borderline, com potencial terapêutico para ambos os sexos. Posto isto, e salientando a importância de agir numa ótica preventiva, o desenvolvimento de uma relação positiva com o eu em idade precoce, por exemplo no início da adolescência, poderá prevenir ou amortecer o desenvolvimento de traços borderline. Face às características da PBP (instabilidade, oscilações entre sentimentos positivos e negativos relativamente ao próprio, elevada impulsividade), a implementação de uma intervenção baseada no desenvolvimento de competências compassivas onde sejam focados aspetos como a autoaversão e a impulsividade, pode ser de grande relevância, quer na população comunitária, quer em amostras de risco (e.g., adolescentes com elevados traços borderline). A autocompaixão tem sido indicada como um processo cognitivoemocional alternativo que envolve outros mecanismos neuro-corticais revelando-se eficaz na regulação emocional e no aumento de comportamentos afiliativos (Gilbert, 2009). Neste sentido, programas de intervenção em grupo para adolescentes focados na autocompaixão, como o Making Friends with Yourself (Bluth et al., 2016), adaptado do Mindful Self-Compassion (Neff \& Germer, 2013), mostraram resultados positivos no desenvolvimento de competências de autocompaixão e de mindfulness e na diminuição dos sintomas ansiosos, depressivos, stress e afeto negativo. De notar ainda que dificuldades relacionadas com a sintomatologia da PBP 
podem ser reportadas a partir dos 11 anos (Zanarini et al., 2006), reforçando a importância de uma abordagem clínica e psicoterapêutica.

Consideramos que o presente estudo apresenta pontos fortes, como o facto de abordar variáveis ainda pouco estudadas na população adolescente portuguesa, recorrendo a uma amostra robusta, permitindo assim identificar importantes pistas para atuar de forma preventiva no desenvolvimento de PBP. Não obstante, reconhecemos também algumas limitações. O facto de ter um desenho transversal não permite determinar relações de causalidade entre as variáveis. Estudos longitudinais são essenciais, no sentido de compreender melhor a trajetória desenvolvimental da PBP, explorando os efeitos a longo prazo. A diferença no tamanho da amostra de rapazes e raparigas para conduzir a análise de regressão deve ser considerada e os resultados interpretados com cautela. Em estudos futuros as diferenças entre rapazes e raparigas relativamente aos traços borderline devem ser profundamente exploradas, com amostras proporcionais, procurando compreender especificidades próprias de ambos os sexos. Também nos parece essencial testar em Portugal a eficácia de programas de intervenção para adolescentes, focados na autocompaixão, como antídoto do autocriticismo e vivência negativa e aversiva com o próprio, promovendo maior autotranquilização (Gilbert, 2009), no sentido de prevenir o agravamento e desenvolvimento de traços borderline.

Agradecimentos | Acknowledgements: Os autores agradecem a todos os Estabelecimentos de Ensino que facilitaram a recolha da amostra, bem como a todos os/as jovens que aceitaram participar na investigação. Os autores agradecem ainda à FCT, pelo financiamento do trabalho.

Conflito de interesses | Conflict of interest: Todos os procedimentos tiveram em conta os padrões éticos do Ministério da Educação e da Comissão Nacional de Proteção de Dados, bem como com a declaração de Helsínquia (1964).

Fontes de financiamento | Funding sources: Este estudo tem o apoio da Bolsa de Doutoramento (SFRH/BD/129985/2017) do primeiro autor, financiada pela Fundação para a Ciência e Tecnologia (FCT).

Contributos: DC: Revisão da literatura, recolha, inserção e tratamento de dados; redação do manuscrito. PC: Contributo na redação do manuscrito e revisão do manuscrito. MC: Contributo na redação do manuscrito e revisão do manuscrito

\section{Referências}

American Psychiatric Association. (2013). Diagnostic and statistical manual of mental disorders (5a ed.). American Psychiatric Association.

Aragonès, E., Salvador-Carulla, L., López-Muntaner, J., Ferrer, M., \& Piñol, J. L. (2013). Registered prevalence of borderline personality disorder in primary care databases. Gaceta Sanitaria, 27(2), $171-174$. https://doi.org/10.1016/j.gaceta.2011.12.006

Bluth, K., \& Blanton, P. W. (2015). The influence of self-compassion on emotional well-being among early and older adolescent males and females. The Journal of Positive Psychology, 10(3), $219-230$. https://doi.org/10.1080/17439760.2014.936967

Bluth, K., Campo, R. A., Futch, W. S., \& Gaylord, S. A. (2017). Age and gender differences in the associations of selfcompassion and emotional well-being in a large adolescent sample. Journal of Youth and Adolescence, 46(4), 840853. https://doi.org/10.1007/s10964-016-0567-2

Bluth, K., Gaylord, S. A., Campo, R. A., Mullarkey, M. C., \& Hobbs, L. (2016). Making friends with yourself: A mixed methods pilot study of a mindful self-compassion program for adolescents. Mindfulness, 7, $479-492$. https://doi.org/10.1007/s12671-015-0476-6 
Bradley, R., Conklin, C. Z., \& Westen, D. (2005). The borderline personality diagnosis in adolescents: Gender differences and subtypes. Journal of Child Psychology and Psychiatry, 46(9), 1006-1019. https://doi.org/10.1111/j.14697610.2004.00401.x

Brown, M. Z., Comtois, K. A., \& Linehan, M. M. (2002). Reasons for suicide attempts and nonsuicidal self-injury in women with borderline personality disorder. Journal of Abnormal Psychology, 111(1), $198-202$. https://doi.org/10.1037//0021-843x.111.1.198

Carreiras, D. (2014). The toxicity of the self: Developing a new measure and testing a comprehensive model of the nature of self-disgust [Tese de mestrado, Universidade de Coimbra]. Repositório Científico da Universidade de Coimbra. https://estudogeral.sib.uc.pt/handle/10316/27653

Carreiras, D., Loureiro, M., Sharp, C., Cunha, C., \& Castilho, P. (2020). Validation of the Borderline Personality Features Scale for Children (BPFS-C) and for Parents (BPFS-P) for the Portuguese population [Manuscrito submetido para publicação]. Faculdade de Psicologia e Ciências da Educação, Universidade de Coimbra.

Carvalho, C. B., Nunes, C., Castilho, P., da Motta, C., Caldeira, S., \& Pinto-Gouveia, J. (2015). Mapping non suicidal selfinjury in adolescence: Development and confirmatory factor analysis of the impulse, self-harm and suicide ideation questionnaire for adolescents (ISSIQ-A). Psychiatry Research, 227(2-3), $238-245$. https://doi.org/10.1016/j.psychres.2015.01.031

Chanen, A. M., Jovev, M., Djaja, D., McDougall, E., Yuen, H. P., Rawlings, D., \& Jackson, H. J. (2008). Screening for borderline personality disorder in outpatient youth. Journal of Personality Disorders, 22(4), 353-364. https://doi.org/10.1521/pedi.2008.22.4.353

Chapman, A. L., Leung, D. W., \& Lynch, T. R. (2008). Impulsivity and emotion dysregulation in borderline personality disorder. Journal of Personality Disorders, 22(2), 148-164. https://doi.org/10.1521/pedi.2008.22.2.148

Cohen, J. (1988). Statistical power analysis for the behavioral sciences (2a ed.). Lawrence Erlbaum Associates.

Crick, N., Murray-Close, D., \& Woods, K. (2005). Borderline personality features in childhood: A short-term longitudinal study. Development and Psychopathology, 17(4), 1051-1070. https://doi.org/10.1017/S0954579405050492

Cross, C. P., Copping, L. T., \& Campbell, A. (2011). Sex differences in impulsivity: A meta-analysis. Psychological Bulletin, 137(1), 97-130. https://doi.org/10.1037/a0021591

Cunha, M. A., Xavier, A., \& Castilho, P. (2016). Understanding self-compassion in adolescents: Validation study of the Self-Compassion Scale. Personality and Individual Diferences, 93, 56-62. https://doi.org/10.1016/j.paid.2015.09.023

Cunha, M., Martinho, M. I., Xavier, A., \& Espírito-Santo, H. (2013). 1405 - Early memories of positive emotions and its relationships to attachment styles, self-compassion and psychopathology in adolescence Resumos do 21th European Congress of Psychiatry, 28(1). https://doi.org/10.1016/S0924-9338(13)76444-7

Dancey, C. P., \& Reidy, J. (2017). Statistics without maths for psychology (7a ed.). Pearson Education.

Ekman, P. (1992). An argument for basic emotions. Cognition and Emotion, 6(3-4), 169-200. https://doi.org/10.1080/02699939208411068

Fossati, A., Gratz, K. L., Maffei, C., \& Borroni, S. (2014). Impulsivity dimensions, emotion dysregulation, and borderline personality disorder features among Italian nonclinical adolescents. Borderline Personality Disorder and Emotion Dysregulation, 1(1), Artigo 5. https://doi.org/10.1186/2051-6673-1-5

Gilbert, P. (2009). Introducing compassion-focused therapy. Advances in Psychiatric Treatment, 15(3), $199-208$. https://doi.org/10.1192/apt.bp.107.005264

Guilherme, M. (2019). Amo ou odeio o meu eu? O impacto da autoaversão na adolescência [Tese de mestrado não publicada]. Universidade de Coimbra.

Guilherme, M., Carreiras, D., Cunha, M., \& Castilho, P. (2020). Validation of the Multidimensional Self-Disgust Scale for adolescents (MSDS-A) [Manuscrito submetido para publicação]. Faculdade de Psicologia e Ciências da Educação, Universidade de Coimbra.

Guiomar, R. N. M. (2015). O impacto da auto-aversão na psicopatologia [Tese de mestrado, Universidade de Coimbra]. Repositório Científico da Universidade de Coimbra. https://estudogeral.sib.uc.pt/handle/10316/31912

Ille, R., Schöggl, H., Kapfhammer, H., Arendasy, M., Sommer, M., \& Schienle, A. (2014). Self-disgust in mental disorders Symptom-related or disorder-specific? Comprehensive Psychiatry, 55(4), 938-943. https://doi.org/10.1016/j.comppsych.2013.12.020

Johnson, J. G., Cohen, P., Kasen, S., Skodol, A. E., \& Oldham, J. M. (2008). Cumulative prevalence of personality disorders between adolescence and adulthood. Acta Psychiatrica Scandinavica, 118(5), $410-413$. https://doi.org/10.1111/j.1600-0447.2008.01231.x

Keng, S. L., \& Wong, Y. Y. (2017). Association among self-compassion, childhood invalidation, and borderline personality disorder symptomatology in a Singaporean sample. Borderline Personality Disorder and Emotion Dysregulation, 4, Artigo 24. https://doi.org/10.1186/s40479-017-0075-3 
Kline, R. (2005). Principles and practice of structural equation modelling (2a ed.). The Guilford Press.

Krawitz, R. (2012). Behavioural treatment of severe chronic self-loathing in people with borderline personality disorder. Part 2: Self-compassion and other interventions. Australasian Psychiatry, 20(6), 501-506. https://doi.org/10.1177/1039856212459586

Krieger, T., Alteinstein, D., Baettig, I., Doerig, N., \& Holtforth, M. G. (2013). Self-compassion in depression: Associations with depressive symptoms, rumination, and avoidance in depressed outpatients. Behavior Therapy, 44(3), 501-513. https://doi.org/10.1016/j.beth.2013.04.004

Leichsenring, F., Leibing, E., Kruse, J., New, A. S., \& Leweke, F. (2011). Borderline personality disorder. Lancet, 377(9759), 74-84. https://doi.org/10.1016/S0140-6736(10)61422-5

Lewinsohn, P. M., Rohde, P., Seeley, J. R., \& Klein, D. N. (1997). Axis II psychopathology as a function of Axis I disorders in childhood and adolescence. Journal of the American Academy of Child \& Adolescent Psychiatry, 36(12), 17521759. https://doi.org/10.1097/00004583-199712000-00024

Loess, P. (2015). Self-compassion as a moderator of the relationship between emotion dysregulation and borderline personality. [Tese de mestrado, Universidade de Montana - Missoula]. Arquivo da Universidade de Montana. https://scholarworks.umt.edu/etd/4526

Lovibond, P. F., \& Lovibond, S. H. (1995). The structure of negative emotional states: Comparison of the Depression Anxiety Stress Scales (DASS) with the Beck Depression and Anxiety Inventories. Behaviour Research and Therapy, 33(3), 335-343. https://doi.org/10.1016/0005-7967(94)00075-u

Marsh, I. C., Chan, S. W. Y., \& MacBeth, A. (2018). Self-compassion and psychological distress in adolescents - A metaanalysis. Mindfulness, 9, 1011-1027. https://doi.org/10.1007/s12671-017-0850-7

Morey, L. C., Warner, M. B., \& Boggs, C. D. (2002). Gender bias in the personality disorders criteria: An investigation of five bias indicators. Journal of Psychopathology and Behavioral Assessment, 24, 55-65. https://doi.org/10.1023/A:1014005308914

Neff, K. (2003). Self-compassion: An alternative conceptualization of a healthy attitude towards oneself. Self and Identity, 2(2), 85-101. https://doi.org/10.1080/15298860309032

Neff, K. D. (2016). The Self-Compassion Scale is a valid and theoretically coherent measure of self-compassion. Mindfulness, 7, 264-274. https://doi.org/10.1007/s12671-015-0479-3

Neff, K. D., \& Germer, C. K. (2013). A pilot study and randomized controlled trial of the Mindful Self-Compassion Program. Journal of Clinical Psychology, 69(1), 28-44. https://doi.org/10.1002/jclp.21923

Neff, K. D., Rude, S. S., \& Kirkpatrick, K. L. (2007). An examination of self-compassion in relation to positive psychological functioning and personality traits. Journal of Research in Personality, 41(4), 908-916. https://doi.org/10.1016/j.jrp.2006.08.002

Olatunji, B. O., David, B., \& Ciesielski, B. G. (2012). Who am I to judge? Self-disgust predicts less punishment of severe transgressions. Emotion, 12(1), 169-173. https://doi.org/10.1037/a0024074

Overton, P. G., Markland, F. E., Taggart, H. S., Bagshaw, G. L., \& Simpson, J. (2008). self-disgust mediates the relationship between dysfunctional cognitions and depressive symptomatology. Emotion, 8(3), 379-385. https://doi.org/10.1037/1528-3542.8.3.379

Pais-Ribeiro, J. L., Honrado, A., \& Leal, I. (2004). Contribuição para o estudo da adaptação portuguesa da Escala de Ansiedade, Depressão e Stress (EADS) de 21 itens de Lovibond e Lovibond. Psicologia, Saúde e Doença, 5(2), 229239. https://bit.ly/2AwXixR

Paris, J. (2009). Borderline personality disorder. In P. H. Blaney \& T. Millon (Eds.) Oxford textbook of psychopathology (2 a ed., pp. 723-737). Oxford University Press.

Plener, P. L., Schumacher, T. S., Munz, L. M., \& Groschwitz, R. C. (2015). The longitudinal course of non-suicidal selfinjury and deliberate self-harm: a systematic review of the literature. Borderline Personality Disorder Emotional Dysregulation, 2. Artigo 2. https://doi.org/10.1186/s40479-014-0024-3

Powell, P. A., Simpson, J., \& Overton, P. G. (2015). An introduction to the revolting self: Self-disgust as an emotion schema. In P. A. Powell, P. G. Overton, \& J. Simpson (Eds.), The revolting self. Perspectives on the psychological, social, and clinical implications of self-directed disgust (pp. 1-24). Karnac Books.

Power, M. J., \& Dalgleish, T. (2008). Cognition and emotion: From order to disorder (2a ed.). Psychology Press.

Scheibner, H. J., Daniels, A., Guendelman, S., Utz, F., \& Bermpohl, F. (2018). Self-compassion mediates the relationship between mindfulness and borderline personality disorder symptoms. Journal of Personality Disorders, 32(6), 838856. https://doi.org/10.1521/pedi_2017_31_331

Schienle, A., Haas-Krammer, A., Schöggl, H., Kapfhammer, H.-P., \& Ille, R. (2013). Altered state and trait disgust in borderline personality disorder. Journal of Nervous \& Mental Disease, 201(2), $105-108$. https://doi.org/10.1097/NMD.0b013e31827f64da 
Sebastian, A., Jacob, G., Lieb, K., \& Tüscher, O. (2013). Impulsivity in borderline personality disorder: A matter of disturbed impulse control or a facet of emotional dysregulation? Current Psychiatry Reports, 15. Artigo 339. https://doi.org/10.1007/s11920-012-0339-y

Sharp, C., \& Bleiberg, E. (2007). Borderline personality disorder in children and adolescents. In A. Martin and F. R., Volkmar, \& M. Lewis (Eds.), Lewis's child and adolescent psychiatry: Comprehensive textbook (4a ed., pp. 680-691). Lippincott Williams and Wilkins.

Sharp, C., Steinberg, L., Temple, L., \& Newlin, E. (2014). An 11-item measure to assess borderline traits in adolescents: Refinement of the BPFSC using IRT. Personality Disorders: Theory, Research, and Treatment, 5(1), 70-78. https://doi.org/10.1037/per0000057

Swartz, M., Blazer, D., George, L., \& Winfield, I. (1990). Estimating the Prevalence of borderline personality disorder in the community. Journal of Personality Disorders, 4(3), 257-272. https://doi.org/10.1521/pedi.1990.4.3.257

Trull, T. J., Jahng, S., Tomko, R. L., Wood, P. K., \& Sher, K. J. (2010). Revised NESARC personality disorder diagnoses: Gender, prevalence, and comorbidity with substance dependence disorders. Journal of Personality Disorders, 24(4), 412-426. https://doi.org/10.1521/pedi.2010.24.4.412

Warren, R. (2015). Commentary on emotional processing in a ten-session general psychiatric treatment for borderline personality disorder: A case study. Personality and Mental Health, 9(1), 84-86. https://doi.org/10.1002/pmh.1290

Xavier, A., Pinto-Gouveia, J., \& Cunha, M. (2016). The protective role of self-compassion on risk factors for non-suicidal self-injury in adolescence. School Mental Health, 8, 476-485. https://doi.org/10.1007/s12310-016-9197-9

Yarnell, L. M., Stafford, R. E., Neff, K. D., Reilly, E. D., Knox, M. C., \& Mullarkey, M. (2015). Meta-analysis of gender differences in self-compassion. Self and Identity, 14(5), 499-520. https://doi.org/10.1080/15298868.2015.1029966

Zanarini, M. C., Frankenburg, F. R., Hennen, J., Reich, D. B., \& Silk, K. R. (2006). Prediction of the 10-year course of borderline personality disorder. American Journal of Psychiatry, 163(5), 827-832. https://doi.org/10.1176/ajp.2006.163.5.827 